\title{
DEUX LETTRES INÉDITES DE PROUDHON
}

\author{
INTRODUCTION
}

Ces deux lettres de Proudhon dont les originaux font partie de la collection proudhonienne de l'Institut International d'Histoire Sociale, se rapportent aux diverses périodes de la vie du grand socialiste français.

1. La première lettre jette une lumière intéressante sur le caractère de Proudhon et sur l'esprit dans lequel celui-ci arrivait à Paris en novembre 1838. Proudhon, qui était alors dans sa trentième année, avait quitté Besançon pour Paris comme „pensionnaire Suard”. La pension Suard consistait en 1500 francs, donnés par l'Académie de Besançon tous les trois ans à celui des jeunes gens de Doubs, dépourvu de fortune qui, au jugement de l'Académie de Besançon, aurait été reconnu le plus apte pour la carrière scientifique. Le 31 mai 1838 Proudhon s'était présenté comme candidat 1), conseillé et patronné par Pérennès, son ancien professeur et le 23 août 1838 l'Académie de Besançon le désignait comme titulaire de la pension. Le secrétaire de l'Académie de Besançon, Pérennès, lui donnait une lettre d'introduction pour l'économiste Joseph Droz (1775-1850), qui lui fut désigné comme tuteur. Cette lettre était conçue comme suit:

„Le nouveau titulaire de la Pension Suard, Mr. Proudhon, part demain pour Paris et je le charge d'autant plus volontiers de la lettre qu'il m'a demandée pour vous, que je connais le généreux intérêt que vous portez d'avance à l'élu de l'académie. In est impatient lui-même de vous remercier du bienveillant patronnage dont vous avez consenti à vous charger à son égard. Il comprend que le bonheur d'entrer en relation avec un homme tel que Mr. Droz n'est pas un des moindres avantages de la Pension Suard. Vous trouverez ses formes

1) La longue lettre de candidature se trouve dans la Correspondance (Paris, 1875, I, p. 24-33). Elle est reproduite dans les Oeuvres completes de Proudhon. Ed. Rivière, Paris 1926, p. 10-16. 
rudes et un peu sauvages. Mais sous cette enveloppe se trouvent d'excellentes qualités et un talent réel. Mr. Proudhon connait toutes les espérances qui reposent sur lui, et partant tous les devoirs que lui impose le choix de l'académie. Il va trouver à Paris ce qu'il a toujours ardemment désiré, des livres et du temps, et tout porte à croire qu'à l'aide des études nouvelles qu'il va faire, et surtout de vos excellents avis, il pourra un jour faire honneur à la franche comté. Veuillez agréer d'avance, Monsieur, et illustre confrère, les remerciements de l'académie pour les soins que vous voulez bien donner à son enfant adoptif." 1)

Tout en étant compositeur et correcteur d'imprimerie à Besançon, Proudhon avait continué ses études linguistiques. En 1837, en réimprimant un livre sur les élements primitifs des langues de l'Abbé Bergier ${ }^{2}$ ), il avait publié en annexe un essai de grammaire générale 3 ).

Ses relations avec son tuteur étaient d'un caractère assez réservé. Il ne se sentait pas à l'aise dans le milieu des académiciens à Paris et il refusait d'aller aux soirées de Droz. Au commencement il rendait souvent visite à ce dernier, il le vit deux fois par semaine, discutait avec lui ses études, mais cependant ne pouvait s'entendre avec lui. Il le regardait comme un honnête homme, plein de bienveillance, mais „,'est bien l'esprit le plus antiphilosophique, le génie le moins scientifique qui fût jamais. D'ailleurs il désespère de moi!" 4)

Le lendemain de sa première visite chez Droz, celui-ci introduisit Proudhon chez Feuillet, bibliothécaire de l'Institut.

1) Lettre inédite de Pérennès à Droz du 11 novembre 1838.

2) Les Elemens primitifs des Langues .... par Bergier. Nouvelle édition augmentée d'un essai de grammaire générale, par l'imprimeurediteur. Besançon, Lambert et Co. imprimeurs 1837. 344 pp.

Une troisième édition a été publiée en $\mathbf{1 8 5 0}$ avec le nom de Proudhon our le titre. Les Elémens primitifs des Langues.... Nouvelle édition augmentée d'un essai de grammaire générale par P.-J. Proudhon. Besançon-Paris, 1850. $344 \mathrm{pp}$.

3) Essai de Grammaire Générale, d'après les principes établis par Bergier. p. 257-339.

4) Lettre à Bergmann du 29 juin, 1840: Correspondance I, p. 218. Une semaine plus tard il écrivit à Droz: „comme pensionnaire Suard, je ne reviendrai pas a Paris. Ainsi, Monsleur, les rapports qui existaient entre nous cessent dès aujourd'hui". 
„C'est à cette recommandation à $M$. Feuillet et à mes visites à sa bibliothèque que se bornent tous les actes de tutelle de M. Droz, et à peu près tous les avantages de mon séjour à Paris", écrivait-il à Pérennès le 9 décembre 1838.

2. Après le coup d'état du 2 décembre Proudhon faisait souvent des démarches en faveur des prisonniers et exilés. II s'adressait aussi à cet effet au cousin de Louis-Napoléon, le prince Napoléon, fils de Jérome Bonaparte, l'ancien roi de Westphalie. C'était le Prince Napoléon qui intervenait en faveur de la requête faite par Proudhon dans sa lettre du 10 août 18521 ).

Dans une lettre du 7 août 1852 Proudhon écrivit à Alexandre Herzen qu'il sollicitait la mise en liberté de ses amis Pilhes et Duchêne, „martyrs désormais inutiles d'une cause perdue en première instance" et il ajoutait: ,je joindrai à ma requête votre nom, mon cher Herzen, et celui de Charles Edmond; comptez sur ma discrétion pour faire les choses à notre convenance et entière satisfaction. L'interdiction de la France est inutile à votre gloire et à notre cause; continuez vos voyages; mais il faut que vous puissiez venir à Paris, car en fin de compte, on n'est libre, on ne pense que là." 2)

Herzen avait fait la connaissance de Proudhon chez Bakounine lors de sa première visite à Paris en 1847. In avait fourni les fonds pour la Voix du Peuple en 1849 et y avait collaboré comme rédacteur. Le 24 avril 1850, quelques semaines avant l'interdiction du journal, Herzen reçut un ordre d'expulsion à cause de sa collaboration à la Voix $d u$ Peuple.

Herzen partit pour Nice le 22 juin. C'est à Nice qu'avaient lieu les catastrophes mentionnées dans la lettre de Proudhon, lorsque dans la nuit du 15 au 16 novembre 1851 Herzen per-

1) Pour les relations entre Proudhon et le prince Napoléon (qui signait Napoléon-Jérome) et pour les opinions de Proudhon au lendemain du coup d'état et pour son attitude à l'égard de Napoléon voir l'Introduction d'Eduard Dolléans et Georges Duveau à La Révolution Sociale dans les Oeuvres Completes de Proudhon, Ed. Rivière, Paris 1936, pp. 7-106.

2) Correspondance IV, p. 317. 
dit sa mère et son fils dans un naufrage. A cet occasion Proudhon écrivit une lettre émouvante à Herzen 1), et quelques mois plus tard Herzen perdait sa femme à la suite d'un accouchement.

Victor Pilhes avait été un membre actif des Sociétés Secrètes sous la Monarchie de juillet et fut nommé commissaire dans le département de l'Ariège dont il fut élu représentant du peuple lors des élections de mai 1849. In avait des relations avec Proudhon depuis 1846 et collaborait avec l'entreprise du „Peuple”. L'agent secret de la police De la Hodde se disait son ami, mais l'espionnait et le dénonçait. Ce serpent vénimeux, écrit Pilhes, ce fantôme sinistre du mouchard De la Hodde me trahissant à chaque heure du jour, durant huit ans, dans ma religion républicaine, dans mon amitié, dans mes intérêts, dans toutes les relations enfin de ma vie intime ${ }^{2}$ ). Quand la trahison de l'agent secret fut découvert c'était Pilhes qui s'opposait à l'exécution du mouchard. Pilhes fut arrêté lors des manifestations du 13 juin 1849 et condamné à la déportation par la Haute Cour de Versailles le 13 octobre.

Georges Duchêne (1824-1876), auteur de „L'Empire Industriel" et autres livres contre la haute finance sous le second Empire et principal auteur du „Manuel du Speculateur à la Bourse" (en collaboration avec Proudhon) avait été rédacteur aux journaux de Proudhon. Il fut condamné comme gérant du „Peuple".

Charles Edmond, pseudonyme de l'écrivain et réfugié polonais Ch. E. Chojecki, auteur de "Souvenirs d'un dépaysé" et beaucoup de pièces de théâtre, était un ami de Herzen et de Proudhon. Il avait été rédacteur de La Voix du Peuple et fut comme Herzen expulsé. Deux jours avant sa libération de trois ans de prison Proudhon lui écrivit de Saint Pélagie le 2 juin 1852: "votre retour d'exile sera une de mes premières occupations".

Au mois d'août on faisait savoir à Proudon, que sa requête

1) Le texte original de cette lettre fut publié pour la première fois par Labry dans son livre „Herzen et Proudhon".

2) Cf. Troisième Lettre à mes amis et a mes concitoyens de l'A riège. Paris, avril 1849 par V. Pilhes. 
venait un peu tôt ${ }^{1}$ ). L'expulsion de Charles Edmond fut cependant annulée quelques mois plus tard 2). Plus tard Proudhon renouvelait sa démarche. Après l'amnistie à l'occasion de la proclamation de I'Empire, Duchêne obtint sa liberté. En mai 1853, grâce à l'intervention du Prince Napoléon, Pilhes fut transféré de Belle-Isle à Sainte-Pélagie. I fut mis en liberté au mois de juillet.

A. L.

Paris, 26 novembre 1838.

Monsieur Droz,

Je me vois dans la nécessité de vous confesser le vrai motif qui m'a empêché de me trouver hier soir à votre réunion, afin que vous ne me supposiez pas une légéreté de conduite ou une grossièreté de moeurs qui ne sont point dans mon coeur. Je me suis rendu d'abord dans la rue de la Chaise dans la ferme résolution de me présenter chez vous: jamais il ne m'a été possible de franchir votre porte. J'ai fait le guet dans la rue pendant une heure, m'approchant quelquefois de la maison, et fuyant comme un malfaiteur dès que j'entendais quelqu'un approcher. J'ai compté les personnes qui me paraissaient se rendre à votre soirée, et à chaque nouvel arrivant je sentais augmenter mes frayeurs. Bref, je m'en suis retourné comme j'étais venu.

Je sens, monsieur, sans qu'il soit besoin qu'on me le dise, tout ce qu'il y a de puérile, de niais dans une pareille timidité; je sais combien cela est de mauvais genre, et peut me nuire dans le monde; je me dis quelquefois qu'il y a peut-être au

1) Correspondance, t. IV, p. 334.

2) Le 28 novembre 1852 Proudhon notait dans son Carnet: „Mardi dernier, 23, retour de Ch. Edmond Choieski par la protection de Napoléon fils de Jérôme." Carnets de Proudhon, publiés par Clément Rocheì dans La Grande Revue 1908, p. 429. 
fond de tout cela plus de vanité et d'amour-propre que de modestie et d'humilité: j'ai beau me prêcher, j'ai beau voir de quel côté sont la raison et les convenances; mon imagination s'effarouche lorsque je veux essayer de me vaincre et lorsque le moment décisif est arrivé, je n'écoute plus que mon épouvante. Oui, je dis bien, je crains les regards et les discours de tout le monde; j'ai peur d'un enfant, d'une femme: je suis embarrassé devant un portier; je suis dans la peine chaque fois qu'il faut demander mon dîner à un restaurant. Ah! monsieur, vous m'avez recommandé de faire l'intrigue; je vous le pardonne de tout mon coeur, mais vous ne me connaissez guère. Avez-vous oublié ce que c'est qu'un franc-comtois pur sang, pure race, qui n'a jamais vécu qu'avec ses rêveries, toujours en contemplation entre le ciel et ses sapins? Je ne suis encore que cela: depuis vingt ans que j'ai l'âge de raison, j'ai presque toujours vécu seul; et vous voudriez me transplanter tout à coup dans la société la plus distinguée! Non, jamais je ne pourrai supporter une telle épreuve. La faute n'en est pas à moi seul peut-être; et j'aurais quelque droit d'en accuser ceux qui m'ont voulu enseigner sur ce que j'avais à faire pour paraître dans le monde: leurs exhortations n'ont fait qu'éveiller mes craintes, en m'instruisant sur ma triste défectuosité.

Je suis honteux de vous révéler toute l'imbécillité de mon âme; mais le mal est fait et je n'y sais plus de remède. Je souhaite comme une faveur précieuse de n'être point violenté dans mes sauvages appréhensions; et je me croirai heureux, si je puis à Paris ne connaître que M. Droz et M. Feuiilet. Je désire surtout n'être désormais presenté ni recommandé à personne: ces deux excellents hommes me suffisent. Vous avez inspection sur moi, monsieur, vous devez surveiller mes études et ma conduite: dites-moi ce que j'ai à faire pour me placer en quelque sorte sous vos regards et votre main. Je souhaite d'être connu de vous, et observé de près.

Je présume que la conversation que vous désiriez avoir avec moi devait rouler sur la direction de mes études: permettezmoi donc avant de finir, de vous en toucher quelque chose. 

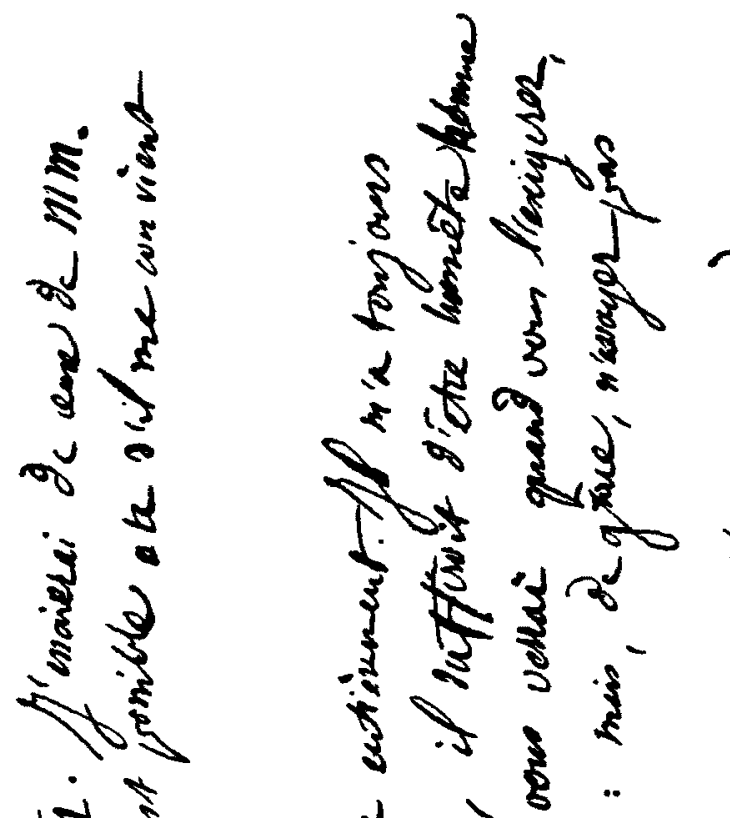

12

15

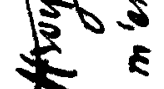

2

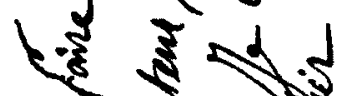

${ }^{d} k$

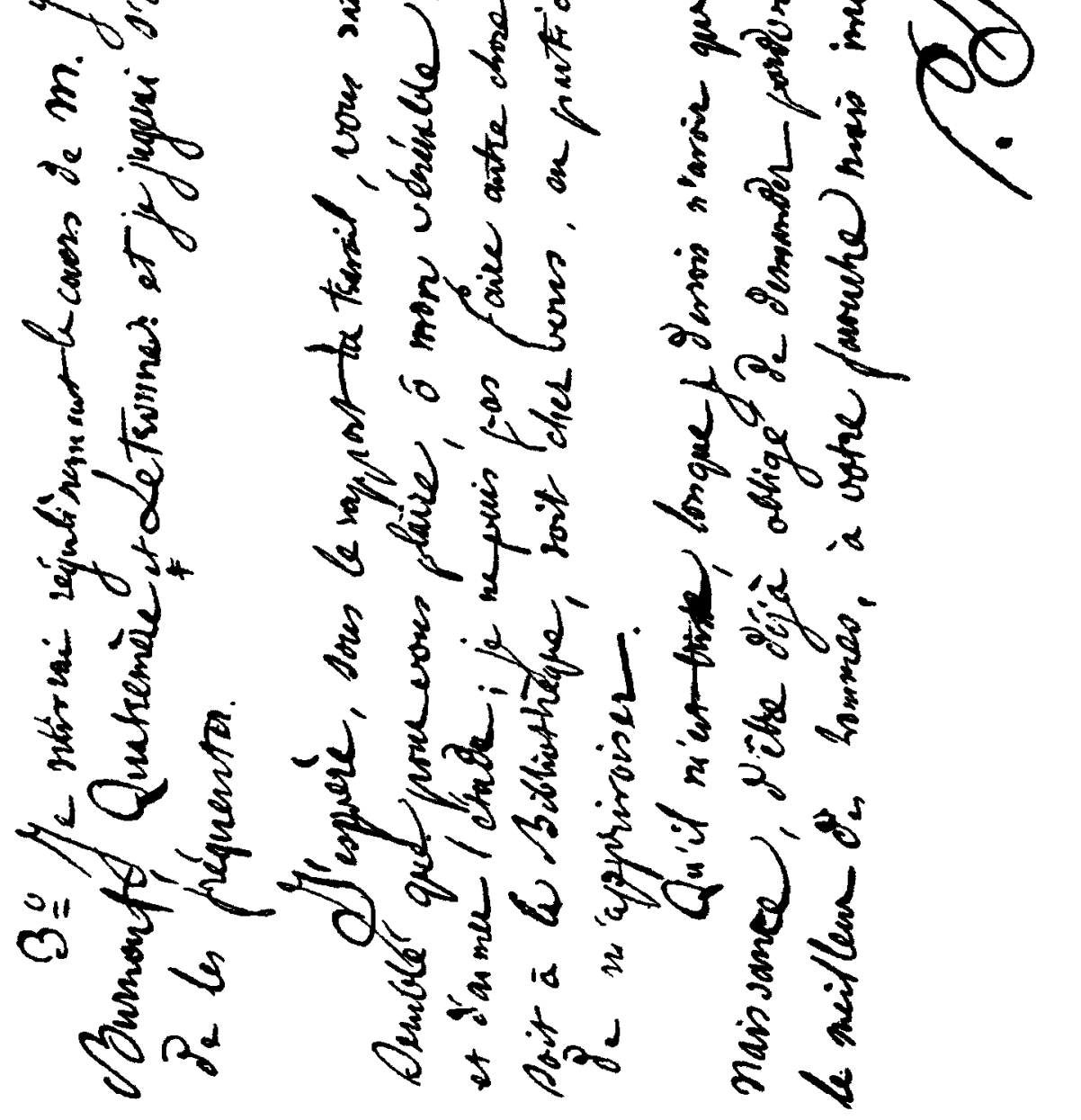

Facsimile de la fin de la lettre de P. J. Proudhon à Joseph Droz de 26 novembre 1838. 
1. J'ai commencé déjà à la Bibliothèque royale et a celle de l'Institut, mes explorations sur le langage, non pas tant dans un but de science et d'érudition, que dans des vues toutes philosophiques. Je considère la parole comme le plus grand et le plus important des phénomènes psychologiques: c'est dans l'espoir d'en obtenir quelques renseignements sur les idées et la morale, que j'ai entrepris l'analyse du fait merveilleux du langage. Je trouve très peu de linguistes et de philologues qui aient observé le langage sous ce point de vue: et ce qu'on a déjà dit n'est presque rien.

2. J'ai commencé à rédiger et classer un certain nombre de notes sur la Bible, que j'avais éparsés sur des chiffons de papier: ces notes m'appartiennent presque toutes. Je suis dans l'intention de faire une Histoire critique des Hébreux, comme il n'en existe pas encore: je suis pour cela une méthoàe différente de celle de la plupart des commentaires; et j'ai cru déjà sur plusieurs points être arrivé à des résultats si naturels, si simples, si lumineux, que je n'abandonnerai le sujet que lorsque je l'aurai entièrement épuisé.

3. Je suivrai régulièrement le cours de M. Jouffroy. J'essaierai de ceux de MM. Burout, Quatremère et Letronne: et je jugerai s'il m'est possible ou s'il me convient de les fréquenter.

J'espère sous le rapport du travail, vous satisfaire entièrement. Il m'a toujours semblé que pour vous plaire, ô mon vénérable tuteur, il suffirait d'être honnête homme et d'aimer l'étude; je ne puis pas faire autre chose. Je vous verrai, quand vous l'exigerez, soit à la Bibliothèque, soit chez vous, en particulier: mais, de grâce, n'essayez pas de m'apprivoiser. Qu'il m'est triste, lorsque je devrais n'avoir que des expressions d'amour et de reconnaissance, d'être déjà obligé de demander pardon d'une esclandre! Pardonnez donc, le meilleur des hommes, à votre farouche mais incorrigible pupille, 
Paris, 10 août 1852.

Monsieur et honoré compatriote,

Vous m'avez laissé espérer que vous pourriez vous intéresser au sort de quelques amis que j'ai dans les prisons et dans l'exil, et qui, partageant d'ailleurs presque en tout mes idées, seraient en mesure d'offrir au pouvoir, en échange de leur liberté, l'assurance de leur résignation pacifique.

Je vous apporte la notice de ces amis, en vous priant de la communiquer à qui de droit, et d'insister sur les observations qui suivent.

Ma démarche n'est point officieuse: elle n'a rien d'ambigu, de honteux, de subreptice. Concertée avec les intéressés, elle est l'expression sincère de leurs résolutions et de leurs idées. Ces amis pensent donc, ils disent et sont prêts à déclarer, qu'à leurs yeux l'événement du 2 décembre a changé la face des choses; qu'après avoir combattu, de tout leur courage, pour la cause démocratique, ils jugent aujourd'hui inutile à leur parti aussi bien qu'à l'Etat la prolongation de leur sacrifice; et qu'en conséquence, hormis le secret des convictions qu'ils gardent en leur âme, ils sont prêts à déposer, vis-a-vis du gouvernement, toute intention, toute vélléité hostile.

En deux mots, destitués de l'action politique par les chances de la lutte, et retenus en prison uniquement pour la surêté du pouvoir établi, ils échangeraient sans scrupule leur destitution forcée contre une volontaire démission.

Tant qu'a subsisté la Constitution de 1848, ils ont cru leur cause protégée par elle, et quoique condamnés et frappés, leur volonté ne se soumettant pas, ils sentaient que leur place était la prison. Après le 2 décembre, leur détention ne leur semble plus qu'une anomalie, et pour le gouvernement qui les retient, et pour leur propre cause. Quelle que soit leur opinion sur les actes du pouvoir actuel, ils laissent à la réflection du pays, à l'enseignement de l'avenir, au patriotisme de leurs concitoyens demeurés libres, le soin de discuter les conditions de l'état politique, et de donner au Président et à ses ministres 
les avertissements, remontrances, requêtes, protestations etc. que leur suggéreront la surêté, le bien-être, et la gloire du Pays.

1. Pilhes, Victor, exreprésentant du peuple, condamné par la Haute-Cour de Versailles à la suite de la manifestation du 13 juin, en ce moment à Belle-Isle.

Pilhes est un des plus braves soldats de la démocratie, à laquelle il a sacrifié sa jeunesse, le gain qu'il faisait dans sa profession de commerçant, et jusqu'à son modeste patrimoine. Combattant au premier rang dans les journées de février, il a compris que les révolutions se faisaient par les idées, non par les armes. Aussi, nommé commissaire du gouvernement dans l'Ariège, son pays natal, il s'y est fait remarquer par une modération ferme, qui lui a valu l'opposition des exaltés, et a fait échouer sa candidature à la Constituante. Il a publié à cette occasion, une brochure, ou profession de foi, très explicite, dont les principes étaient, pour l'époque, d'une remarquable sagesse. Au 13 juin 1849, quand une fraction de la Montagne, obéissant à des injonctions maladroites ou perfides, essaya d'exécuter la menace tombée de la tribune, Pilhes ne crut pas devoir se séparer de son chef parlementaire: il fut arrêté au Conservatoire, et condamné à la déportation.

La vie politique de Pilhes est un tissu de dévouements, recompensés des plus odieuses ingratitudes. C'est à lui que le fameux de la Hodde s'était attaché comme son ombre, sous le règne de Louis-Philippe; c'est Pilhes qui, lorsque ce même de la Hodde fut mis en jugement par une espèce de conseil de guerre montagnard, sauva la vie au traître, et empêcha un meurtre que la morale et la politique eussent avoué, mais que l'esprit général de modération à cette époque réprouvait. $\mathbf{A}$ cette heure d'indignation suprême, Pilhes ne put oublier qu'il avait aimé, pendant trois ans, crédité, soutenu, de la Hodde, comme son frère et son compagnon. Pilhes est dans l'intention, s'il recouvra sa liberté, de rentrer dans les affaires et de reprendre sa carrière commerciale, étranger à la politique et dévoué seulement au travail et à la patrie.

2. Duchêne, Georges, gérant du Peuple et rédacteur de la Voix du Peuple condamné à 29 ans de prison, pour divers délits de presse, en ce moment a Belle-Isle. 
Duchêne est compatriote de Paul-Louis Courier et de Rabelais, dont il a deployé maintefois, dans le Peuple et 'la Voix du Peuple l'originalité, la verve, et l'ironie. Ses articles étaient recherchés, de toutes les classes de lecteurs, avec une avidité extraordinaire. L'ancien préfet de police, M. Carlier, qui eut l'occasion d'apprécier ce jeune homme, pourrait donner sur lui les plus utiles renseignements.

Duchêne, fils d'un charron, charron lui-même, puis compositeur d'imprimerie, puis correcteur, puis écrivain, à présent maître d'école à Belle-Isle, s'est tenu constamment, avec Pilhes, loin des deux partis qui divisent les détenus. Son désir le plus ardent est de revoir sa vieille mère, dont il est le fils unique, et de reprendre, avec ses fonctions industrielles, ses études littéraires, en gardant vis-a-vis du pouvoir de LouisNapoléon la plus stricte neutralité.

A Pilhes et Duchêne, je joins ici les noms de MM. de Herzen et Ch. Edm. Choïeki, le premier russe, le second polonais, tous deux expulsés de France, comme étrangers, pour leurs opinions. M. Herzen, libre penseur, noble coeur, auteur de quelques écrits sur la Russie, remarquables par leur originalité et leur profondeur, bien éloigné des petites conspirations et des intrigues de parti, est un de ces philosophes cosmopolites que la politique des états devrait entretenir pour la synthèse générale des croyances, des idées, des intérêts, et des moeurs. Son expulsion de France est une de ces erreurs de police, inévitables dans les moments de trouble, mais que tout pouvoir fort et stable doit tenir à honneur de réparer. Eprouvé récemment par d'affreux malheurs, après avoir perdu, dans une suite de catastrophes, mère, fils et femme, $M$. de Herzen se proposerait de venir à Paris soigner l'éducation de ces deux jeunes filles, seul adoucissement à ses infortunes. M. Choïeski, ancien rédacteur de la Tribune des Peuples et de la Voix du Peuple est déjà naturalisé français. Sans égard au droit que lui assurait cette naturalisation, la police l'a expulsé, à la suite du vote de la loi du 31 mai, contre la Voix du Peuple, s'était signalée par sa protestation, et dont le maintien a déterminé le coup d'état du 2 décembre. Le fait accompli ayant justifié la prévision de l'écrivain, M. Choieshi peut 
dire qu'après tout il ne demande qu'à partager avec LouisNapaléon le hénéfice de la victoire.

Je crois, Monsieur, que la mise en liberté de Pilhes et Duchêne, ainsi que le rappel de MM. de Herzen et Choieki, dans les termes et aux conditions ci-dessus exprimées, serait, pour le pouvoir, pour le pays, et pour l'émigration, d'un effet moral salutaire, et d'un excellent enseignement.

Je crois surtout qu'il importe à la politique du Président et à ses tendances populaires, de ne pas laisser croire qu'il n'a que des excuses pour les bannis de l'orléanisme, tandis qu'il fait peser l'exil et la chaîne sur ceux de la république. Plein de confiance en vos bons offices, je vous recommande de nouveau mes pauvres amis, et vous prie d'agréer, monsieur et compatriote, l'assurance de mes sentiments les plus profonds et de mon plus respectueux hommage.

P. J. PROUDHON 